Article

\title{
Characteristics of BeiDou-3 Experimental Satellite Clocks
}

\author{
Yifei Lv ${ }^{1}$, Tao Geng ${ }^{1,2, *(D)}$, Qile Zhao ${ }^{1,2}$ and Jingnan Liu ${ }^{1}$ \\ 1 GNSS Research Center, Wuhan University, Wuhan 430079, China; lvyifei@whu.edu.cn (Y.L.); \\ zhaoql@whu.edu.cn (Q.Z.); jnliu@whu.edu.cn (J.L.) \\ 2 Collaborative Innovation Center of Geospatial Technology, Wuhan 430079, China \\ * Correspondence: gt_gengtao@whu.edu.cn; Tel.: +86-27-6877-8767
}

Received: 30 September 2018; Accepted: 19 November 2018; Published: 22 November 2018

check for updates

\begin{abstract}
The characteristics of the improved Atomic Frequency Standard (AFS) operated on the latest BeiDou-3 experimental satellites are analyzed from day-of-year (DOY) 254 to 281, of the year 2017, considering the following three aspects: stability, periodicity, and prediction precision. The two-step method of Precise Orbit Determination (POD) is used to obtain the precise clock offsets. We presented the stability of such new clocks and studied the influence of the uneven distribution of the ground stations on the stability performance of the clock. The results show that the orbit influence on the Medium Earth Orbit (MEO) clock offsets is the largest of three satellite types, especially from $3 \times 10^{3} \mathrm{~s}$ to $8.64 \times 10^{4} \mathrm{~s}$. Considering this orbit influence, the analysis shows that the Passive Hydrogen Maser (PHM) clock carried on C32 is approximately $2.6 \times 10^{-14}$ at an interval of $10^{4}$, and has the best stability for any averaging intervals among the BeiDou satellite clocks, which currently achieves a level comparable to that of the PHM clock of Galileo, and the rubidium (Rb) clocks of Global Positioning System (GPS) Block IIF. The stability of the improved Rb AFS on BeiDou-3 is also superior to that of BeiDou-2 from $3 \times 10^{2} \mathrm{~s}$ to $3 \times 10^{3} \mathrm{~s}$, and comparable to that of Rb AFS on the Galileo. Moreover, the periodicity of the PHM clock and the improved Rb clock are presented. For the PHM clock, the amplitudes are obviously reduced, while the new Rb clocks did not show a visible improvement, which will need further analysis in the future. As expected, the precision of the short-term clock prediction is improved because of the better characteristics of AFS. The Root Mean Square (RMS) of 1-h clock prediction is less than $0.16 \mathrm{~ns}$.
\end{abstract}

Keywords: BeiDou-3; clock analysis; Allan variance; harmonic analysis; clock prediction

\section{Introduction}

The BeiDou-2 constellation presently consists of 14 active satellites and can be grouped into the following three types: five Geostationary Orbit (GEO) satellites, six Inclined Geostationary Orbit (IGSO) satellites, and three Medium Earth Orbit (MEO) satellites [1]. All of them are operated on $\mathrm{Rb}$ Atomic Frequency Standard (AFS) [2]. According to the three-step strategy, the BeiDou-3 that was under construction was planned to complete the constellation consisting of 35 satellites, and to provide global service for users around the world [1,3,4]. Therefore, by the end of February 2016, China had launched five experimental satellites for validating the BeiDou-3 performance, including two IGSOs (C31 and C32) and three MEOs (C33, C34, and C35). Unfortunately, the signal of C35 cannot be obtained by the ground receivers because of technical reasons [5].

The new generation of experiment satellites have several improvements over the older BeiDou-2 satellite [6]. First, it can broadcast new types of signals, B1C (1575.42 MHz) [7], B2a (1176.45 MHz) [8], and $\mathrm{B} 2 \mathrm{~b}$, to improve the compatibility and interoperability with other navigation satellite systems. Second, the BeiDou-3 experiment satellites are equipped with the improved rubidium ( $\mathrm{Rb})$ AFS 
(C31, C33, and C34) and the Passive Hydrogen Maser (PHM) (C32 and C35) produced by China's own technology. Third, Inter-Satellite Links (ISL), one of the most important ingredients for global BeiDou system design, is used to enable autonomous navigation. The detailed information of the BeiDou-3 experiment satellites is shown in Table 1.

Table 1. On-broad clock type of BeiDou-3 experimental satellites on 1 October 2017. PHM-passive hydrogen maser.

\begin{tabular}{cccc}
\hline Pseudo Random Noise (PRN) & Type & Launch Date & Clock \\
\hline C31 & IGSO01-S & 2015.03 .30 & $\mathrm{Rb}$ \\
C32 & IGSO02-S & 2015.09 .30 & PHM \\
C33 & MEO01-S & 2015.07 .25 & $\mathrm{Rb}$ \\
C34 & MEO02-S & 2015.07 .25 & $\mathrm{Rb}$ \\
C35 & MEO03-S & 2016.02 .01 & PHM \\
\hline
\end{tabular}

With the above-mentioned characteristics in mind, researchers have been able to further their studies. Chen et al. confirmed that ISL contributes enormously to the space signal accuracy, in particular, when the satellites are not tracked by a regional network [6]. Zhang et al. presented an initial assessment of the new-generation signals and of the initial results of single epoch relative positioning [9]. Tan et al. showed the initial results of the precise orbit determination with nine stations [10]. Xie et al. evaluated the multipath effect and the performance of the precise orbit and clock determination [11]. Zhao, et al. proved that the best orbit determination solution for BeiDou-3e is obtained with five-parameter Extended CODE Orbit Model (ECOM) and a three-day arc [12]. Wu et al. elaborated on a brief introduction of PHM design, and also analyzed the performance of both PHM and the improved Rb AFS on-board clock [13].

Currently, there are two main methods to estimate the precision satellite clock offsets, called Two-Way Satellite Time and Frequency Transfer (TWSTFT), and Orbit Determination and Time Synchronization (ODTS). The BeiDou official clock products in the broadcast ephemeris are estimated by the former method [14,15], and the performance of the BeiDou-3 experimental clock that was obtained in this way has been fully discussed in the literature [13]. However, this method has some drawbacks. The clock offsets obtained by TWSTFT are calculated independently without orbit information, and as a result, they are not coupled with the orbit solution like the ODTS method is. For this reason, the high-precision clock products obtained by ODTS are the main products, such as the International Global Navigation Satellite System (GNSS) Service (IGS) products, that are used by general users. Therefore, the characteristics of such clock products need to be further studied.

This paper focuses on the characteristics of the improved Rb AFS as well as the PHM deployed by the BeiDou-3 experiment satellites. The precise clock products are obtained though the strategy of two-step method of orbit determination. The stabilities of the onboard clocks of both the BeiDou-3 and BeiDou-2 satellites, as well as GPS, GLObal NAvigation Satellite System (GLONASS), and Galileo satellites are evaluated using the Overlapping Allan Deviation (OADEV). In view of the limited number of tracking stations for BeiDou-3, a controlled trial using the current BeiDou-2 satellites is set up to figure out the impact of the performance of the clock offsets estimated by global stations and regional stations. Moreover, the frequency characteristics of the BeiDou-3 satellites are discussed with Non-Uniform Fast Fourier Transform (NUFFT), and the results of the prediction are presented in order to verify the clock characterization.

\section{Data Collection}

The BeiDou clock offset is one of the products of the Precise Orbit Determination (POD) calculated from a modified version of the Positioning and Navigation Data Analyst (PANDA) software developed by Wuhan University, China [16]. International GNSS Monitoring and Assessment System (iGMAS) [17] and IGS Multi-GNSS Experiment (MGEX) tracking network stations are used to collect 
BeiDou-3 observations for the time span day-of-year 254 to 281, of the year 2017. The distribution of the tracking stations is shown in Figure 1. The green dots represent the selected global stations, which can be capable of tracking the BeiDou-2 satellites. The red dots represent the regional stations, which are mainly distributed in the Asia Pacific region and have access to the BeiDou-3 signal. As a result, there is a loss percentage from about $11 \%$ to $15 \%$ of the BeiDou-3 MEO satellites in the calculated clock offsets, because of the insufficient measurements.

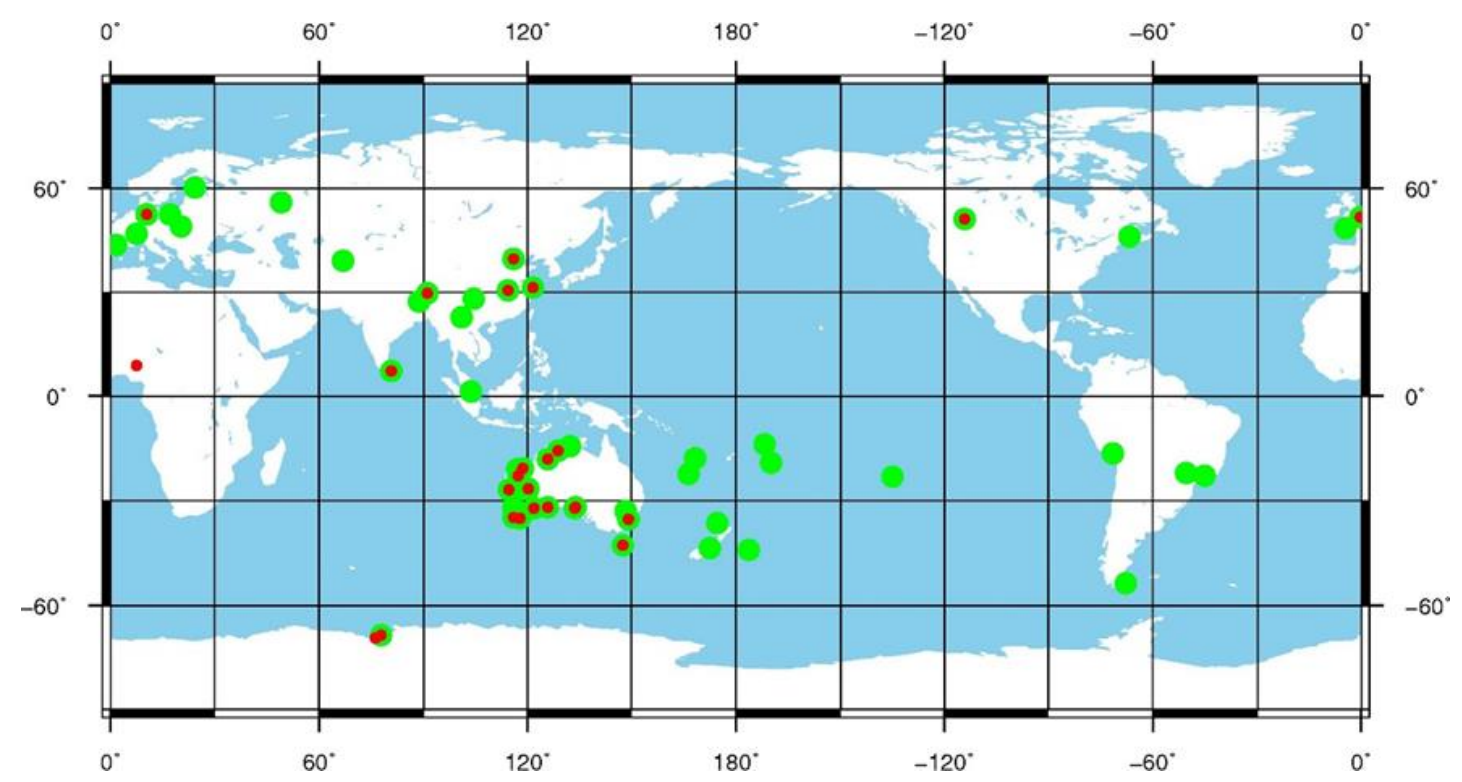

Figure 1. Distribution of the tracking stations used in this study. Stations capable of tracking BeiDou-3 observations are marked in red. The other stations are marked in green.

We calculated the global and regional clock offsets from the global stations and regional stations, respectively. If not explicitly stated, the global clock offsets have been selected for the following analyses. Note that the red stations can only receive and identify the B1 and B3 signal both adopted in BeiDou-2 and BeiDou-3. Therefore, the following characteristics of the BeiDou clocks are analyzed based on the B1 and B3 signal.

The two-step method to determinate the BeiDou orbit and clock could be described as follows: To begin with, the parameters that are same as those of the BeiDou system, such as the station coordinates, receiver clocks, and tropospheric delay, are estimated from the GPS observations using precise point positioning. Then, these parameters are fixed so as to determine the BeiDou satellite orbit and clock $[18,19]$. The Phase Center Offset (PCO) and Phase Center Variation (PCV) correction provided by Wuhan University are used for BeiDou-2 satellites [20], while only the PCO correction provided by the Operation Control Department (OCD) is used for the BeiDou-3 experiment satellites. The details of the POD processing strategy can be found in Table 2.

Table 2. Processing strategy for the POD.

\begin{tabular}{ll}
\hline \multicolumn{1}{c}{ Correction } & \multicolumn{1}{c}{ Model } \\
\hline Observation type & Code and carrier observations of B1 and B3 \\
Measurements combination & Ionosphere-free combination \\
Elevation cutoff & $10^{\circ}$ \\
Sampling rate & $300 \mathrm{~s}$ \\
Weight & A priori precision of $0.002 \mathrm{~m}$ and $0.2 \mathrm{~m}$ for phase and code observables, and \\
& elevation-dependent weighting factor \\
Station coordinates & Estimated in step one and fixed in step two \\
Receiver clocks & Estimated in step one and fixed in step two \\
Inter system biases & Estimated in step two \\
\hline
\end{tabular}


Table 2. Cont.

\begin{tabular}{ll}
\hline \multicolumn{1}{c}{ Correction } & \multicolumn{1}{c}{ Model } \\
\hline Tropospheric delay & Saastamoinen model [21], global mapping function [22], and two-hourly \\
& $\begin{array}{l}\text { Zenith Tropospheric Delays (ZTD) without gradients, which are obtained in } \\
\text { step one and fixed in step two }\end{array}$ \\
Phase wind up & Corrected [23] \\
Tide displacement & Solid Earth tide, pole tide, and ocean tide loading; according to \\
& International Earth Rotation Service (IERS) Conventions 2010 model [24] \\
Relativity effect & IERS Conventions 2010 model [24] \\
Geopotential & EIGEN_GL04C up to 12 $\times$ 12 degrees and orders \\
N-body gravitation & Jet Propulsion Laboratory (JPL) DE405 ephemeris model \\
Solar radiation & ECOM five-parameter model [25] \\
\hline
\end{tabular}

Because there are no precise clock products for the experimental satellites, the overlapping arcs of the three-day POD solutions are used to evaluate the precision of the so-called twice difference method [26], which can be expressed as follows:

$$
\begin{aligned}
& C(i)=\frac{1}{m} \sum_{s=1}^{m} \delta t_{2}^{s}(i)-\delta t_{1}^{s}(i) \\
& \Delta^{s}(i)=\delta t_{2}^{s}(i)-\delta t_{1}^{s}(i)-C(i) \\
& \mathrm{SD}=\sqrt{\frac{1}{n} \sum_{i=1}^{n}\left(\Delta^{s}(i)-\overline{\Delta^{s}}\right)^{2}}
\end{aligned}
$$

where $\delta t_{1}^{s}(i)$ and $\delta t_{2}^{s}(i)$ are the two clock offsets of the overlapping arcs of satellites, $s$, at the epoch, $i$, and $\Delta^{s}(i)$ is the difference of the clock offset after eliminating the average bias $C(i)$ calculated by $m$ satellites between these two overlapping arcs. The Standard Deviation (SD) of $n$ epochs is computed from Equation (3), where $\overline{\Delta^{s}}$ is the mean of $\Delta^{s}$. The precision of the two-day overlapping clock offsets calculated by two consecutive three-day solutions is shown in Figure 2. Compared with the result from the regional stations, the precision of the BeiDou-2 MEO satellites estimated by the global stations has visibly improved from $40 \%$ to $60 \%$. Therefore, it can be inferred that in the future, the precision of C33 and C34 will be gradually improved through establishing more tracking stations and optimizing the ISL technology.

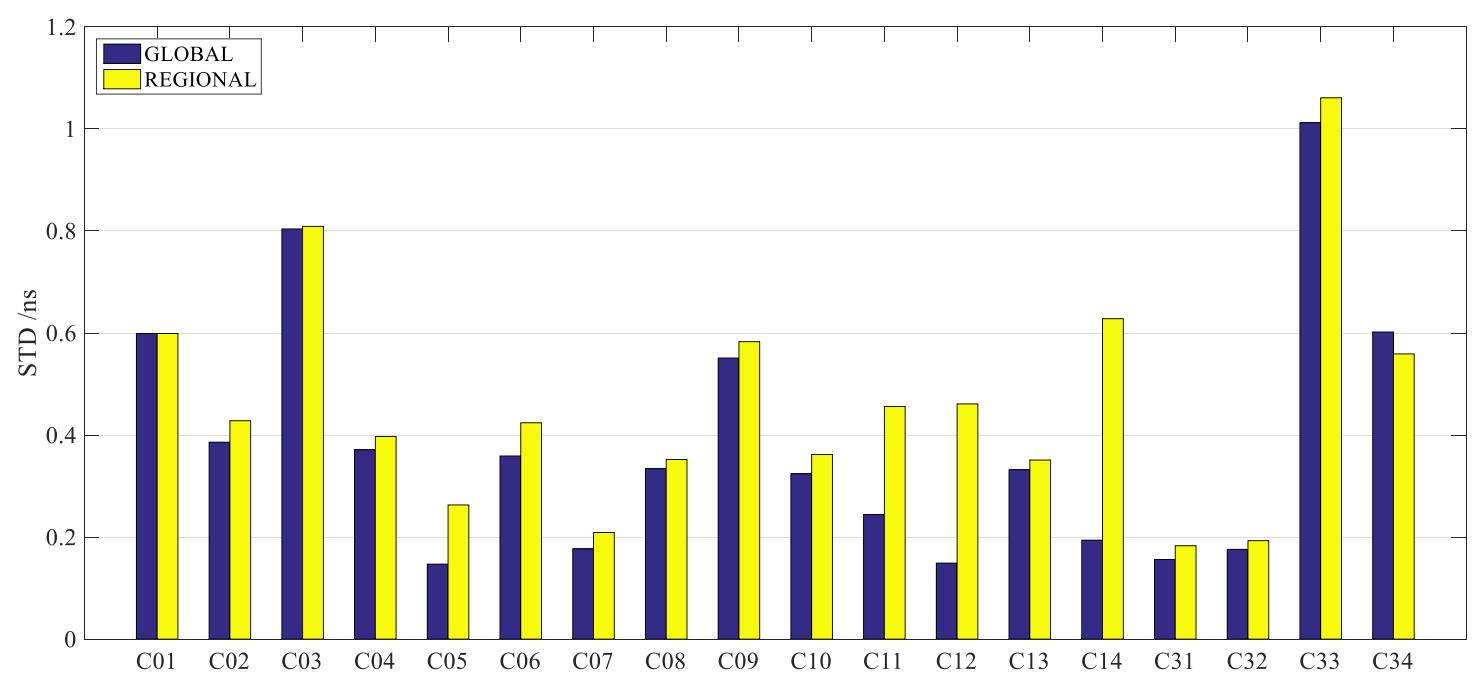

Figure 2. Standard deviation (SD) of two-day clock offsets of two consecutive three-day solutions. 


\section{Pre-Processing for Clock Offset}

The pre-processing quality of the original clock offsets determines the reliability of the on-board clock characteristics. In general, there are three types of anomalies in a set of raw clock offsets, which are the outliers, jumps, and gaps. For the outliers and jumps, the median detection method is applied. First, the phase data in the time domain is converted into frequency data. Second, for the obvious frequency drifts of the BeiDou clocks (shown below), from the authors' experience, the clock offsets are segmented into a single day, and the frequency data of each day is individually detected by the median method.
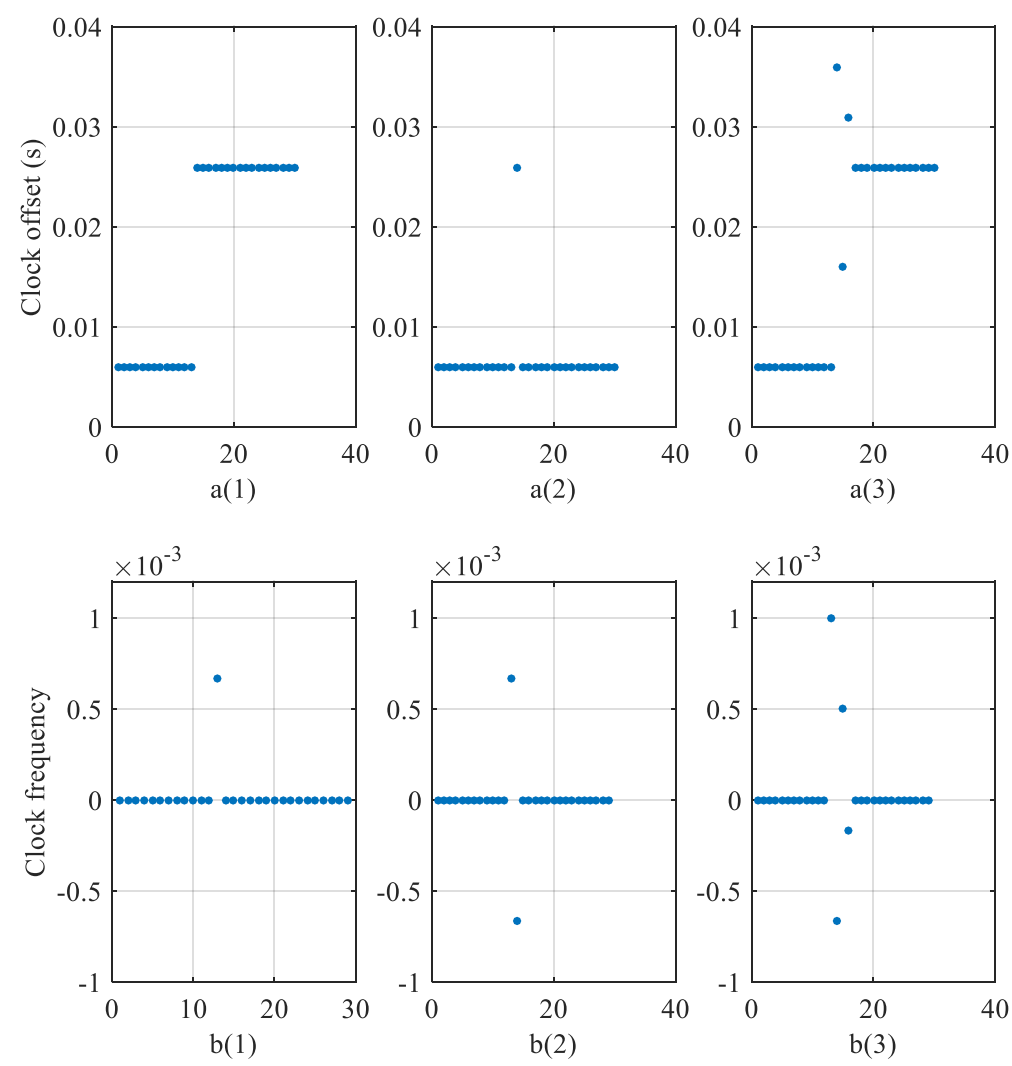

Figure 3. Three types of anomalies in the original clock offsets, $b(1), b(2)$, and $b(3)$, are the frequency sequences corresponding to the clock time series of $\mathrm{a}(1)$, $\mathrm{a}(2)$, and $\mathrm{a}(3)$, respectively.

A deviation limit in terms of the median absolute deviation (MAD) is described as follows [27],

$$
M A D=\operatorname{Median}\left(\frac{\left|y_{i}-\operatorname{Median}\left(y_{i}\right)\right|}{0.6745}\right)
$$

where $y_{i}$ is the clock frequency series, Median $\left(y_{i}\right)$ is the median value of $y_{i}$, and the factor 0.6745 makes the MAD equal to the standard deviation for the normally distributed data. Here, a three times MAD is adopted as a deviation limit for judgment. The anomalies-jumps, outliers, and mixed jumps and outliers-in the original clock offsets are plotted in Figure 3. It can be the concluded that one outlier of the frequency demonstrates a clock jump in the phase sequence, two outliers signify that there is a phase outlier in the time domain, and three or more outliers indicate that there may be the potential for both clock offset jumps and outliers. We found no difficulty in detecting and removing the clock offset outlier shown in a(2) as well as fixing the clock offset jump shown in a(1) with a linear fitting. As for the mixed cases of a(3), three clock offset outliers related to the four frequency outliers shown in $b(3)$ were detected and removed. Thereafter, we recalculated the new frequency sequence and determined whether there was a need to fix the clock jump, like for a(1). The time series of the clock frequency 
after pre-processing is plotted in Figure 4. The frequency amplitudes of the experimental satellites clocks are smaller than those of the BeiDou-2 satellites, and the obvious abnormalities of C04 result in the poor stability reflected in the following analysis.

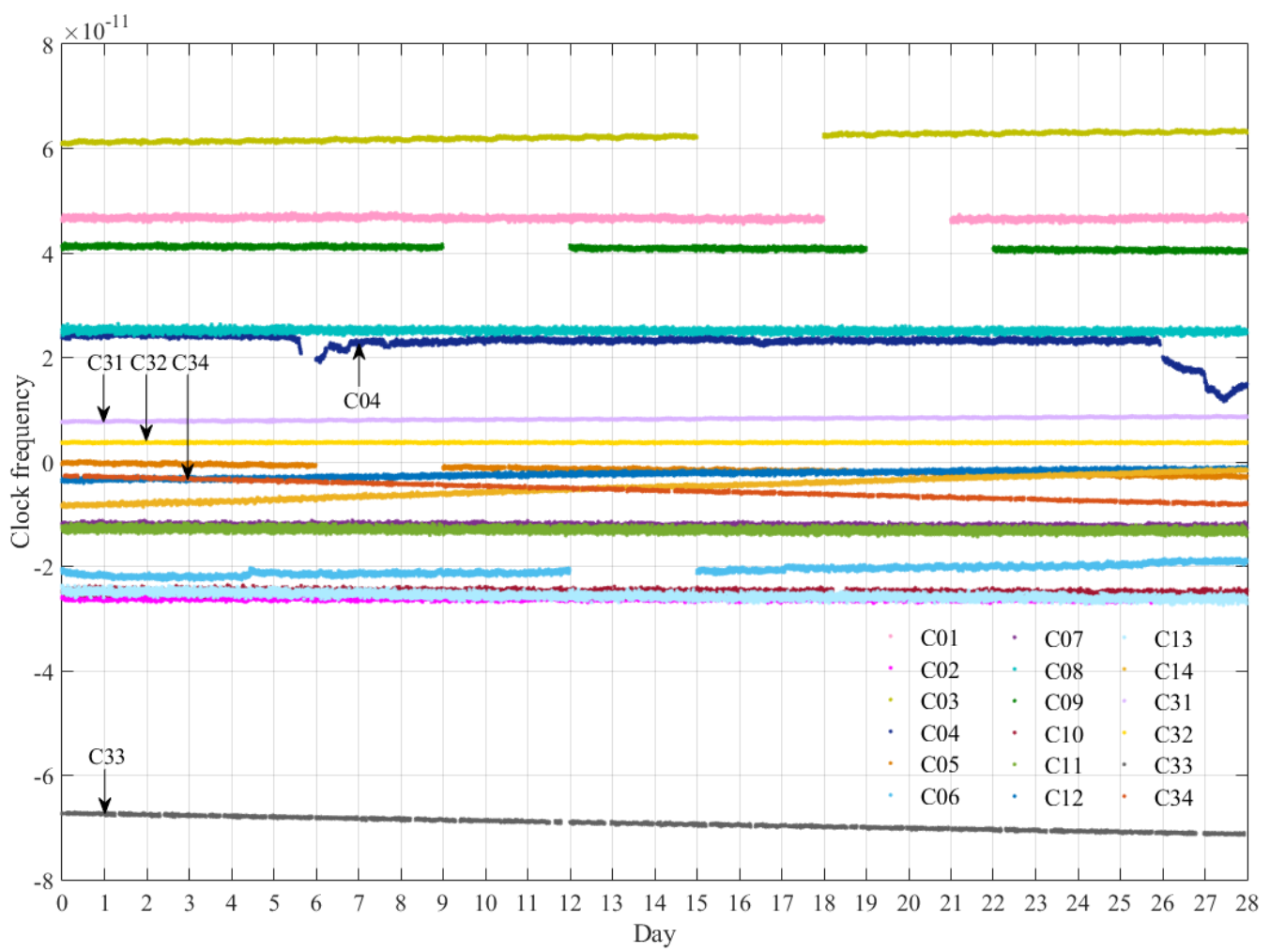

Figure 4. Time series of BeiDou clock frequency after pre-processing varies from day-of-year (DOY) 254 to 281 , of the year 2017 .

\section{Stability Analysis}

The stability of the on-broad clock is critical to the quality of the GNSS measurements, influencing the application of navigation system. To this end, the OADEV is used to characterize the stability of the new PHM and the improved Rb AFS operated on the BeiDou-3 [27]. We refer to the computing strategy proposed in the literature [28], so that it can better reflect the stability of the clock sequence with the missing data. In terms of the phase data $x_{i}$, it can be estimated from a set of $\mathrm{N}$ time measurements, as follows:

$$
\sigma_{y}(\tau)=\sqrt{\frac{1}{2(N-2 m) \tau^{2}} \sum_{i=1}^{N-2 m}\left[x_{i+2 m}-2 x_{i+m}+x_{i}\right]^{2}}, \quad x_{i+2 m} \neq 0, x_{i+m} \neq 0, x_{i} \neq 0
$$

where $\tau=m \tau_{0}$ is the averaging time interval, $m$ is the averaging factor, and $\tau_{0}$ is the basic interval. All of these clock offset sequences have been detrended by removing a daily quadratic trend. In the following discussion, we evaluated the stability of the BeiDou-3 experimental satellites. Then, we analyzed the estimated clock offsets based on the BeiDou-2 global stations and regional stations. After that, the OADEVs of GPS, GLONASS, and Galileo were compared in order to evaluate the frequency stability of the new AFS carried on the BeiDou-3.

\subsection{Comparison with Regional Solution}

The OADEVs of the BeiDou regional and global clock offsets are shown in Figure 5. The four plots in Figure 5, from left to right and from top to bottom, are the OADEV graphs of GEO, IGSO, MEO, and 
BeiDou-3, respectively. The subscripts " $G$ " and " $R$ " in the legends represent the global solution and regional solution. The two auxiliary lines, $1 / \sqrt{\tau}$ and $1 / \tau$, correspond to the white phase noise and white frequency noise, respectively.
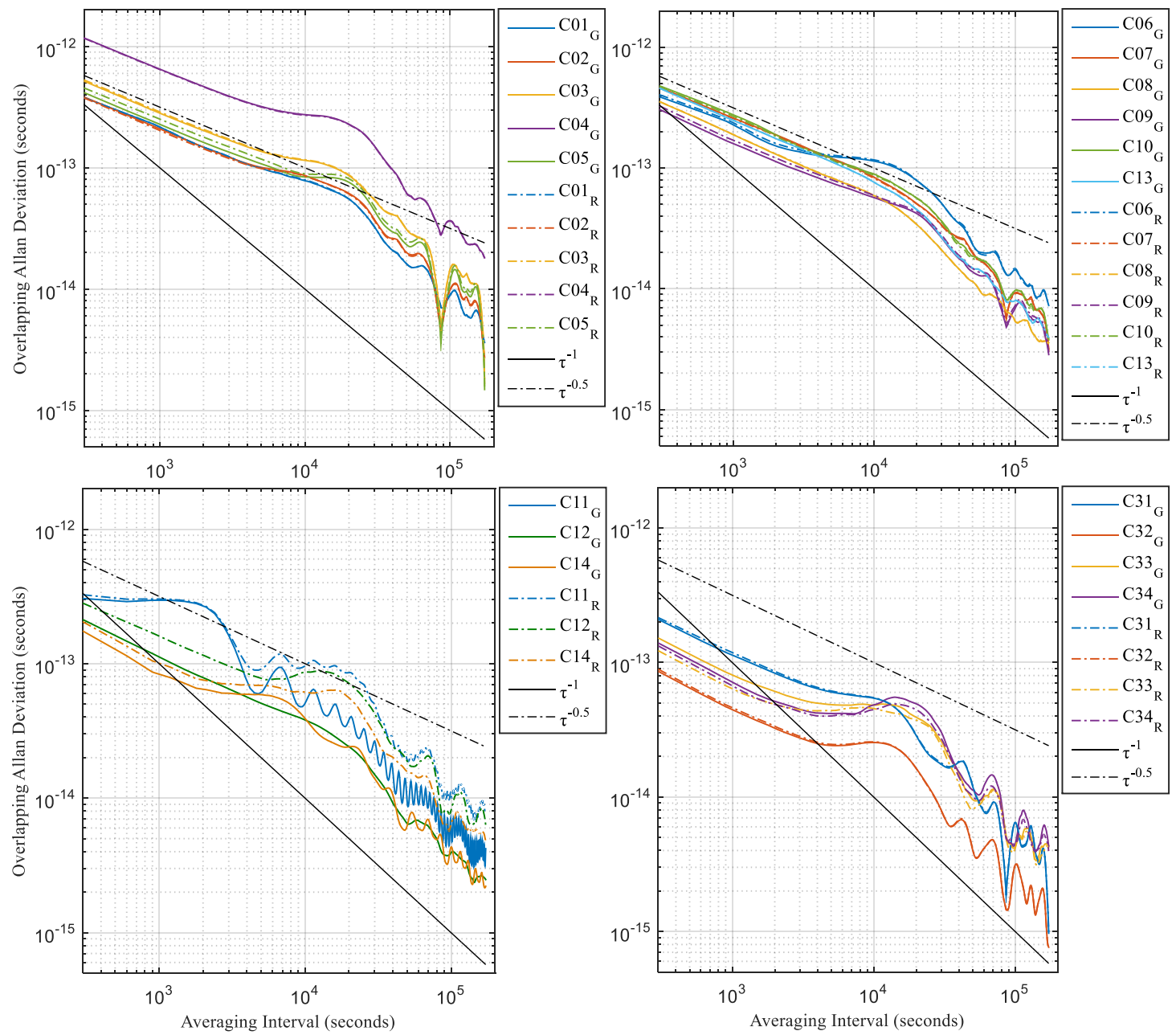

Figure 5. Overlapping Allan Deviation (OADEV) comparison of BeiDou regional and global clock offsets.

As it is depicted in the bottom right plot, the stability for the experimental satellites of the global solution are similar to that of the regional solution, because of using of the same tracking stations, (i.e., the red stations plotted in Figure 1). For the new PHM carried on C32, the two distinctly sharp minima that occurred near the $8.64 \times 10^{4} \mathrm{~s}$ and $1.728 \times 10^{5}$ clearly reflect the presence of a 24 -h period signal. Between $0 \mathrm{~s}$ and $8.64 \times 10^{4} \mathrm{~s}$, there are two other steep minima that may be caused by the signal superposition of the 12-h and 8-h cycles related to the orbit period, according to the detail frequency analysis in the latter part. The frequency stability of C32 is approximately $2.6 \times 10^{-14}$ at an interval of $10^{4}$, and $1.4 \times 10^{-15}$ at interval of $8.64 \times 10^{4}$. Nevertheless, C32 equipped with PHM has undoubtedly the lowest OADEV for any of the averaging intervals. The improved Rb AFS on C31 shows a better performance than the corresponding BeiDou-2 IGSO satellites. The rest of the MEO satellites (C33 and C34) that are equipped with improved Rb clocks are also superior to BeiDou-2 in the interval of $3 \times 10^{2}$ $\mathrm{s}$ to $3 \times 10^{3} \mathrm{~s}$. The OADEVs of the BeiDou- 3 clock show a white phase noise behavior from averaging intervals of $3 \times 10^{2} \mathrm{~s}$ to $3 \times 10^{3} \mathrm{~s}$. This is a good supplement to the stability analysis of the TWSTFT method in this interval range, where the real clock stability is buried in the K-band measurement noise [13].

Furthermore, according to Figure 5, for BeiDou-2, the number and distribution of the tracking stations have an impact on the estimated OADEV of the different types of satellites. Especially, the 
bumps shown in MEO regional clock offsets are notably flattened from $3 \times 10^{3} \mathrm{~s}$ to $8.64 \times 10^{4} \mathrm{~s}$ in global clock offsets. Considering that the orbital error affects the stability between $10^{3} \mathrm{~s}$ and $10^{5} \mathrm{~s}$ [14], we believe that the stability of the BeiDou-3 MEO satellites shown in the figure should be different from the actual results. That is, the curve at $10^{4} \mathrm{~s}$ should be smoother like the BeiDou- 2 global solution in bottom left plot. In the same way, the bumps of the BeiDou-3 IGSO satellites at the interval of $10^{4} \mathrm{~s}$ are larger than that of the BeiDou-2 IGSO satellites. Therefore, the clock stability of the BeiDou-3 experimental satellites is more affected by the orbit error because of the limited number and the uneven distribution of the stations.

In addition, there are some anomalies in Figure 5. The frequency abnormalities in Figure 4 can explain the poor stability of C04. The abnormal fluctuation of $\mathrm{C} 11$ is consistent with the literature [12,29], and the bump here is smoother than that from the literature [12].

\subsection{Comparison with Other Global Navigation Systems}

In order to compare the frequency stability with the GPS, GLONASS, and Galileo, the OADEV of each of them has been presented with a time span from DOY 254 to DOY 281, of the year 2017. The IGS final product has been selected for estimating the OADEV of the GPS, and the GBM final product has been selected for GLONASS and Galileo. The satellites chosen here represent the general performance of this type of satellite or atomic clock. The results of the Allan deviation are depicted in the Figure 6 .
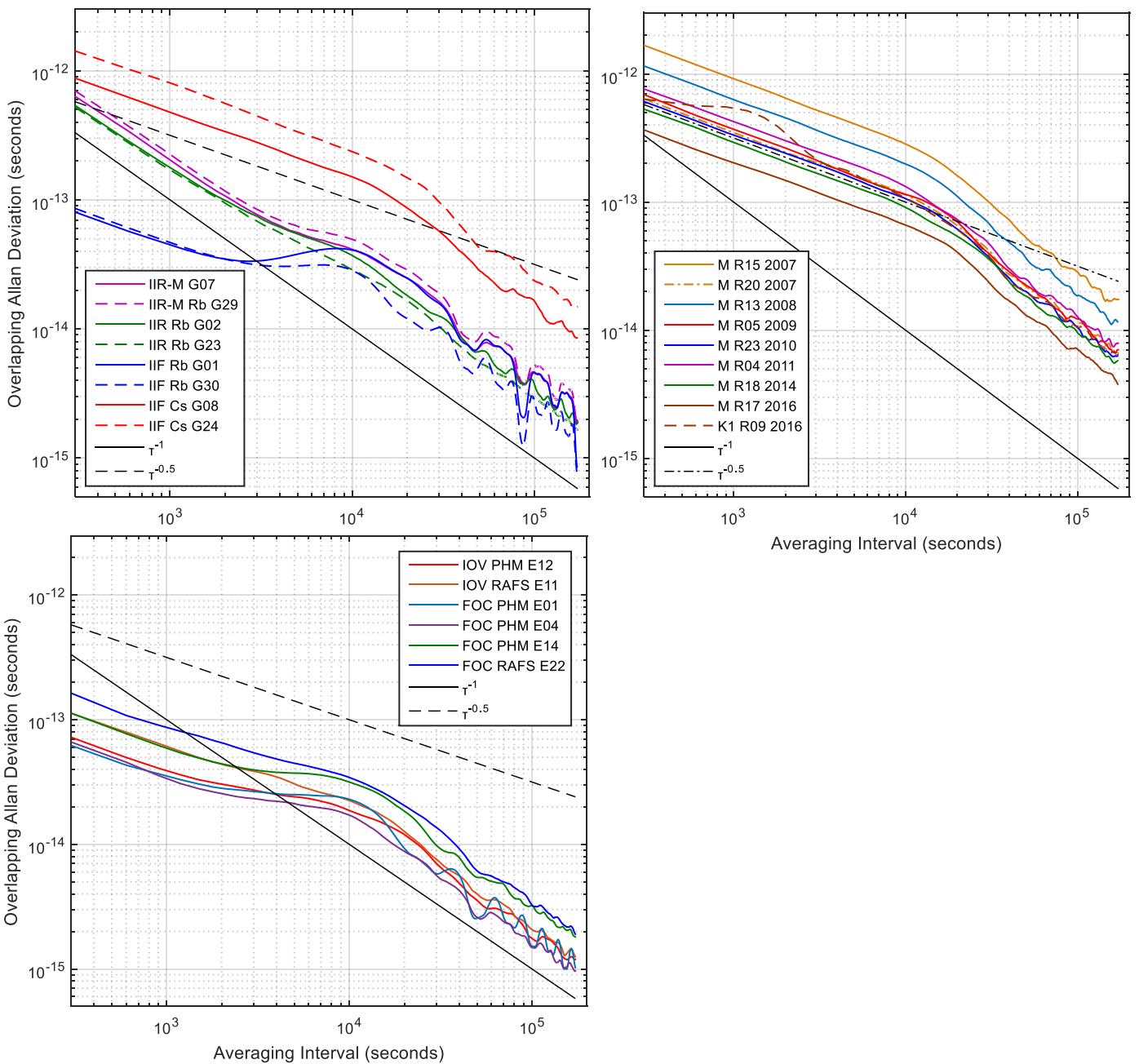

Averaging Interval (seconds)

Figure 6. OADEV of Global Positioning System (GPS), GLObal NAvigation Satellite System (GLONASS), and Galileo from DOY 254 to 281, of the year 2017, with intervals of 300 s. 
Although the pronounced bumps stand out at an average interval of $1.1 \times 10^{4} \mathrm{~s}$, it is not difficult to find that the Rb AFS on Block IIF has a competitive performance among the PHM clock of BeiDou and Galileo. However, in spite of the worse performance, between $300 \mathrm{~s}$ and $1 \times 10^{4} \mathrm{~s}$, the old $\mathrm{Rb}$ clocks on Block IIR and Block IIR-M have no obvious fluctuations and are more stable than Block IIF between the interval of $10^{4} \mathrm{~s}$ and $10^{5} \mathrm{~s}$.

Galileo has launched many satellites since 2015, so E01 and E04, launched in 2016, have been chosen to assess the AFS on these new satellites. From the bottom left plot, we can see that whether FOC or IOV satellites show excellent stability of PHM. The stability of most PHM is superior to that of Rb AFS, except for some satellites, such as E08 shown in figure. Moreover, there is no significant difference between PHM clock performance on new satellites and old satellites. The OADEVs of PHM are less than $2 \times 10^{-14} \mathrm{~s}$ at averaging time of $10^{4} \mathrm{~s}$ and smoother than that of BeiDou-3 PHM and GPS Block IIF Rb AFS, leading to a better clock prediction accuracy in broadcast applications. Compared with Galileo PHM, the BeiDou PHM has a more obvious no-power-law behavior near $1.05 \times 10^{4} \mathrm{~s}$, which results from the orbit error.

The majority of the GLONASS constellation currently consists of GLONASS-M satellites with a Cesium (Cs) clock [30], but the Russia government has started to launch the enhanced GLONASS-K series to gradually replace the existing satellites. As we can see from Figure 6, there is a correlation between the frequency stability and satellite lifetime. The stability of the atomic clock of most of the GLONASS satellites with shorter service times is better than that of the atomic clock of satellites with long service times [31]. On the other hand, the frequency standard equipped on GLONASS-K1 is worse than that of the old GLONASS-M series. Further studies on these new series of satellites are required in order to explain the specific characteristics. The current performance of the GLONASS clock is consistent with BeiDou-2, and better than that of the GPS Cs clock, regardless of life expectancy.

For the above analysis, it follows that the new AFS operated on BeiDou-3 has greatly improved over the old frequency standard adopted by BeiDou-2, especially at the interval from $3 \times 10^{2} \mathrm{~s}$ to $3 \times 10^{3} \mathrm{~s}$. The new PHM currently achieves a level of stability comparable to that of the PHM on Galileo and to the Rb clocks on GPS Block IIF. Considering the orbital error affects due to the limited number and uneven distribution of tracking stations, the stability of the improved Rb AFS on the BeiDou-3 experimental satellites is comparable to that of the Rb AFS on Galileo, and better than that of the rest GPS and GLONASS clocks.

\section{Frequency Spectrum Analysis}

NUFFT are used to further study the periodicity in the residual sequence with gaps [32,33]. An open source code downloaded from the literature [34] is used. Figure 7 shows the amplitude spectrum BeiDou-3 of the new atomic clock frequency standard as well as the BeiDou-2 Rb frequency standard computed by the global clock solution. The horizontal axis represents the cycles per revolution (CPR).

Figure 7 shows the amplitude spectra of the GEO (top), IGSO (middle), and MEO (bottom) satellite clocks. The GEO satellites have the largest frequency amplitude. There are two stronger harmonics, 24-h (1 CPR) and 12-h (2 CPR), and two lesser harmonics, 8-h (3 CPR) and 6-h (4 CPR) in the top plot. The spectrogram of $\mathrm{C} 04$ is as complex as expected, and its performance is consistent with the frequency variation shown in Figure 4.

For the IGSO satellites, the amplitude spectrum of the PHM clock carried on C32 is visibly smaller than that of the $\mathrm{Rb}$ clock carried on the others IGSO satellites. According to the existing research [29], the IGSO satellites of BeiDou-2 have three obvious cycles (i.e., 24-h (1 CPR), 12-h (2 CPR), and 8-h (3 CPR)). However, there is no obvious $24-\mathrm{h}$ period in C32 detected by NUFFT. Presently, it is hard to explain this phenomenon with insufficient measurements of BeiDou-3. On the other hand, C31 shows two strong harmonics of 24-h and 8-h, and a lesser harmonic of 12-h, similar to the BeiDou-2 IGSO satellites. 
The bottom plot of Figure 7 shows that there are common periods of MEO satellites around 12.9-h (1 CPR) and 6.45-h (2 CPR), and the magnitudes are smaller than that of GEO and IGSO. Also, we found that C33 and C34 are noisier than the other MEO satellites, and they have a unique period around 0.92 CPR. Combined with the stability analysis in Figure 6, inadequate measurements may lead to these results.

The periodicity of the clock is closely related to the orbital error. Furthermore, an obvious period of C11 appears near $10.05 \mathrm{CPR}$, similar to the results of the authors of [12]. For the spectrum analysis results obtained by NUFFT, the PHM clock has a great improvement, while the new Rb clock did not show a marked improvement, which needs further evaluation and analysis using increasing tracking stations in the future.

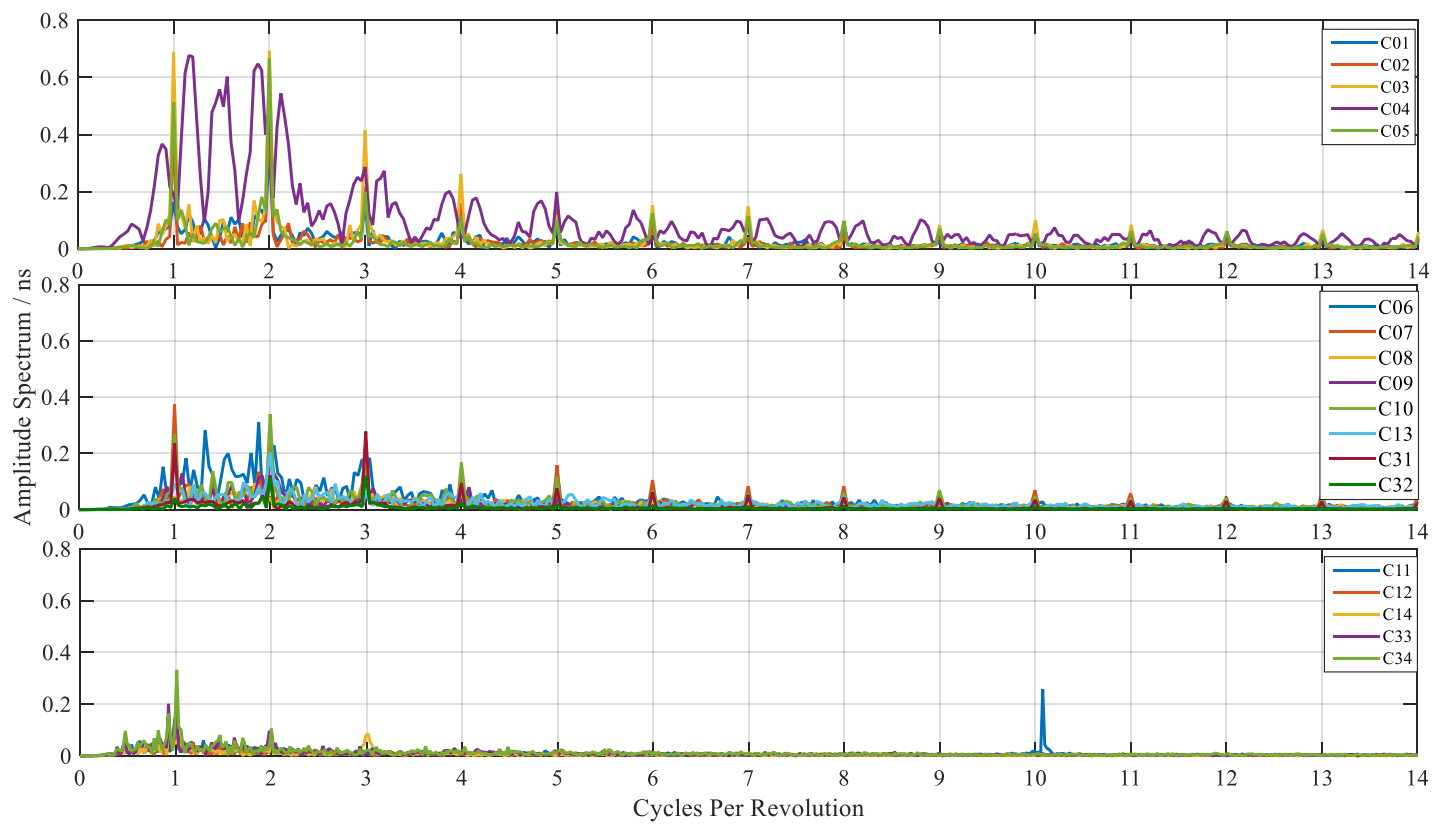

Figure 7. Spectrum analysis obtained by the non-uniform fast Fourier transforms (NUFFT) for BeiDou-2 and BeiDou-3.

\section{Short-Term Clock Prediction Analysis}

The short-term clock prediction results are presented here to further evaluate the stability of the BeiDou-3 experiment satellite clocks. It has a significant meaning, considering the uneven distribution of the tracking stations around the world. Once the satellites run out of sight of the ground stations, the parameters of the orbit and clock will be predicted using the precise ephemeris. In this part, a simple linear model is selected for testing the accuracy of the 1-hour prediction with a sliding window of two hours of precise clock offsets. The previous analysis of periodicity is not considered in this short-term prediction.

The median value and the interquartile range (IQR) of the prediction residuals are shown in Figure 8. Thanks to the new AFS applied on experimental satellites, the IQR of C32 is less than 0.1 ns, and the IQR of C31, C33, and C34 are less than $0.2 \mathrm{~ns}$. These results mean that the degree of dispersion of the new atomic clock prediction results is distinctly improved compared with the old ones on BeiDou-2. For instance, the best performing C14 in the BeiDou-2 satellite is more than twice as much as C32. The precision of the sliding linear clock prediction is listed in Table 3. The Root Mean Square (RMS)of the new PHM and the improved Rb AFS is superior to $0.1 \mathrm{~ns}$ and $0.16 \mathrm{~ns}$, respectively, better than that of BeiDou-2 satellites. The improvement of the stability of the new AFS does improve the precision of the clock prediction. 


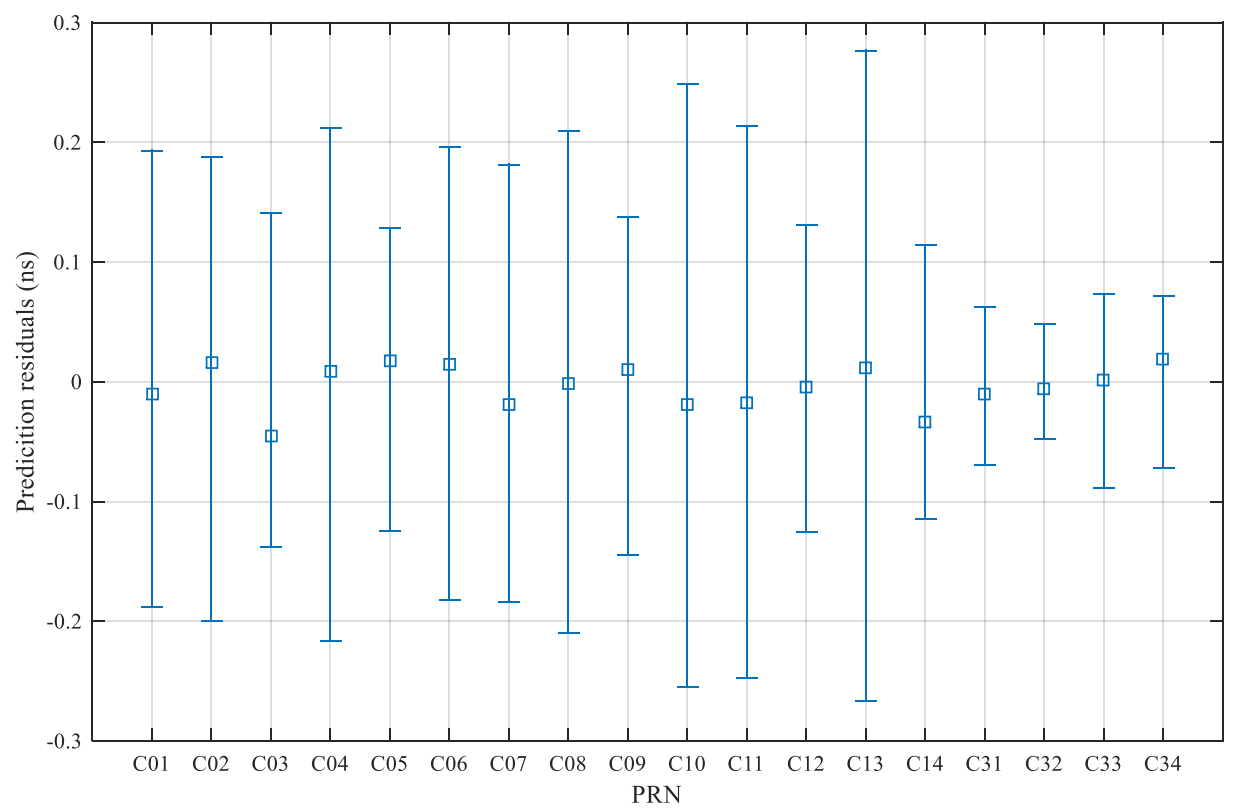

Figure 8. Median (squares) and interquartile range (IQR) (vertical bars) of the residuals of clock prediction.

Table 3. Precision of 1-h sliding linear prediction using 2-h clock offsets.

\begin{tabular}{cccccc}
\hline PRN & Mean (ns) & RMS (ns) & PRN & Mean (ns) & RMS (ns) \\
\hline C01 & -0.013 & 0.321 & C10 & -0.016 & 0.404 \\
C02 & 0.024 & 0.314 & C11 & 0.002 & 0.334 \\
C03 & -0.044 & 0.242 & C12 & -0.011 & 0.212 \\
C04 & 0.015 & 0.452 & C13 & 0.008 & 0.449 \\
C05 & 0.015 & 0.209 & C14 & -0.040 & 0.241 \\
C06 & -0.010 & 0.373 & C31 & -0.006 & 0.104 \\
C07 & -0.018 & 0.305 & C32 & -0.005 & 0.081 \\
C08 & 0.000 & 0.339 & C33 & 0.017 & 0.157 \\
C09 & 0.014 & 0.232 & C34 & 0.018 & 0.124 \\
\hline
\end{tabular}

\section{Conclusions}

In this paper, we focus on the characteristics of the improved AFS operated on the latest BeiDou-3 experimental satellites. The two-step method of precise orbit determination is used to obtain the precise clock offsets. Because of the low quality of the measurements caused by the small number of tracking stations, a pre-processing method is necessarily applied to check the original clock offsets.

The stability of such new clocks is presented and compared with that of GPS, GLONASS, and Galileo. Through the analysis of the clock stability computed from the regional and global stations, we found that subject to the influence of the orbital errors because of the limited number and uneven distribution of the tracking stations, the stability $3 \times 10^{3} \mathrm{~s}$ to $8.64 \times 10^{4} \mathrm{~s}$ fluctuates greatly and does not reflect the real stability of the atomic clock. The results based on this show that the PHM clock carried on C32 is approximately $2.6 \times 10^{-14}$ at interval of $10^{4}$, and has the best stability for any averaging intervals among the BeiDou satellite clocks. Furthermore, the rest of the improved Rb AFS on BeiDou-3 is also superior to the BeiDou-2 at interval of $300 \mathrm{~s}$ to $3 \times 10^{3} \mathrm{~s}$. For comparison with other navigation systems, the PHM on the BeiDou- 3 currently achieves a level of stability comparable to that of the PHM clock of the Galileo and the Rb clocks of the GPS Block IIF, and the improved Rb AFS on BeiDou-3 is comparable to that of the Rb AFS on Galileo, and better than that of the rest of the satellite clocks.

The analysis of NUFFT indicated that the PHM clock has a great improvement. The amplitude of 12-h (2 CPR) and 8-h (3 CPR) periods is obviously reduced compared with other BeiDou-2 IGSO satellites, and there is no obvious $24-\mathrm{h}$ (1 CPR) period. However, the new Rb clock did not show a 
substantial improvement. The performance of this new $\mathrm{Rb}$ frequency needs further evaluation and analysis in the future.

The improvement of the clock stability and frequency periods finally contribute to the precision of the short-term clock prediction. For the BeiDou-3 satellites, the RMS of 1-h prediction is less than $0.16 \mathrm{~ns}$.

As expected, the improved $\mathrm{Rb}$ clocks and PHM clock operated on BeiDou-3 show. a better performance than the old BeiDou-2 clocks. The only regret is that there are too few stations that can received the $\mathrm{B} 1$ and $\mathrm{B} 3$ signal of the experimental satellites. Considering that new types of signals, B1C, $\mathrm{B} 2 \mathrm{a}$, and B2b, are added in BeiDou-3, there is still a lot of work to do with the clock characteristics.

Author Contributions: Y.L. performed the experiments and wrote the paper; T.G. designed the experiments and contributed precise POD product; Q.Z. and J.L. supervised and provided the guiding idea for this study.

Funding: This work is supported by the National Nature Science Foundation of China (no. 41674004 and no. 41574030).

Acknowledgments: International GNSS Monitoring and Assessment System (iGMAS) and IGS Multi-GNSS Experiment (MGEX) are greatly acknowledged for providing BeiDou data.

Conflicts of Interest: The authors declare no conflict of interest.

\section{References}

1. The State Council Information Office of the People's Republic of China. China's BeiDou Navigation Satellite System. Available online: http:/ / www.BeiDou.gov.cn/xt/gfxz/201712/P020171221333863515306. pdf (accessed on 17 April 2018).

2. Han, C.; Yang, Y.; Cai, Z. BeiDou Navigation Satellite System and its time scales. Metrologia 2011, 48, S213-S218. [CrossRef]

3. China Satellite Navigation Office. BeiDou Navigation Satellite System Signal in Space Interface Control Document Open Service Signal (Version 2.1). Available online: http:/ / www.beidou.gov.cn/xt/gfxz/201805/ P020180507527106075323.pdf (accessed on 17 April 2018).

4. Yang, Y.; Li, J.; Xu, J.; Tang, J.; Guo, H.; He, H. Contribution of the Compass satellite navigation system to global PNT users. Chin. Sci. Bull. 2011, 56, 2813-2819. [CrossRef]

5. Zhou, R.; Hu, Z.; Zhao, Q.; Li, P.; Wang, W.; He, C.; Cai, C.; Pan, Z. Elevation-dependent pseudorange variation characteristics analysis for the new-generation BeiDou satellite navigation system. GPS Solut. 2018, 22, 60. [CrossRef]

6. Chen, J.; Hu, X.; Tang, C.; Zhou, S.; Guo, R.; Pan, J.; Li, R.; Zhu, L. Orbit determination and time synchronization for new-generation BeiDou satellites: Preliminary results. Sci. Sin. Phys. Mech. Astron. 2016, $46,1-11$.

7. China Satellite Navigation Office. BeiDou Navigation Satellite System Signal in Space Interface Control Document Open Service Signal B1C (Version 1.0). Available online: http:/ / www.BeiDou.gov.cn/xt/gfxz/ 201712/P020171226741342013031.pdf (accessed on 17 April 2018).

8. China Satellite Navigation Office. BeiDou Navigation Satellite System Signal in Space Interface Control Document Open Service Signal B2a (Version 1.0). Available online: http: / / www.BeiDou.gov.cn/xt/gfxz/ 201712/P020171226742357364174.pdf (accessed on 17 April 2018).

9. Zhang, X.; Wu, M.; Liu, W.; Li, X.; Yu, S.; Lu, C.; Wickert, J. Initial assessment of the COMPASS/BeiDou-3: New-generation navigation signals. J. Geodesy 2017, 91, 1225. [CrossRef]

10. Tan, B.; Tan, B.; Yuan, Y.; Yuan, Y.; Wen, M.; Wen, M.; Ning, Y.; Ning, Y.; Liu, X.; Liu, X. Initial Results of the Precise Orbit Determination for the New-Generation BeiDou Satellites (BeiDou-3) Based on the iGMAS Network. ISPRS Int. J. Geo-Inf. 2016, 5, 196. [CrossRef]

11. Xie, X.; Geng, T.; Zhao, Q.; Liu, J.; Wang, B. Performance of BDS-3: Measurement Quality Analysis, Precise Orbit and Clock Determination. Sensors 2017, 17, 1233. [CrossRef] [PubMed]

12. Zhao, Q.; Wang, C.; Guo, J.; Wang, B.; Liu, J. Precise orbit and clock determination for BeiDou-3 experimental satellites with yaw attitude analysis. GPS Solut. 2017, 22, 367. [CrossRef]

13. Wu, Z.; Zhou, S.; Hu, X.; Liu, L.; Shuai, T.; Xie, Y.; Tang, C.; Pan, J.; Zhu, L.; Chang, Z. Performance of the BDS3 experimental satellite passive hydrogen maser. GPS Solut. 2018, 22, 647. [CrossRef] 
14. Zhou, S.; Cao, Y.; Zhou, J.; Hu, X.; Tang, C.; Liu, L.; Guo, R.; He, F.; Chen, J.; Wu, B. Positioning accuracy assessment for the 4GEO/5IGSO/2MEO constellation of COMPASS. Sci. China Phys. Mech. Astron. 2012, 55, 2290. [CrossRef]

15. Zhou, S.; Hu, X.; Wu, B.; Liu, L.; Qu, W.; Guo, R.; He, F.; Cao, Y.; Wu, X.; Zhu, L.; et al. Orbit determination and time synchronization for a GEO/IGSO satellite navigation constellation with regional tracking network. Sci. China Phys. Mech. Astron. 2011, 54, 1089. [CrossRef]

16. Geng, T.; Xie, X.; Zhao, Q.; Liu, X.; Liu, J. Improving BDS integer ambiguity resolution using satellite-induced code bias correction for precise orbit determination. GPS Solut. 2017, 21, 1191. [CrossRef]

17. Jiao, W. International GNSS Monitoring and Assessment System (iGMAS) and latest progress. In Proceedings of the 2014 China Satellite Navigation Conference (CSNC), Nanjing, China, 21-23 May 2014.

18. Hackel, S.; Steigenberger, P.; Hugentobler, U.; Uhlemann, M.; Montenbruck, O. Galileo orbit determination using combined GNSS and SLR observations. GPS Solut. 2014, 19, 15-25. [CrossRef]

19. Lou, Y.; Liu, Y.; Shi, C.; Wang, B.; Yao, X.; Zheng, F. Precise orbit determination of BeiDou constellation: Method comparison. GPS Solut. 2015, 20, 259-268. [CrossRef]

20. Guo, J.; Xu, X.; Zhao, Q.; Liu, J. Precise orbit determination for quad-constellation satellites at Wuhan University: Strategy, result validation, and comparison. J. Geodesy 2016, 90, 143-159. [CrossRef]

21. Saastamoinen, J. Atmospheric Correction for the Troposphere and Stratosphere in Radio Ranging Satellites. Use Artif. Satell. Geodesy 1972, 15, 247-251.

22. Boehm, J.; Niell, A.; Tregoning, P.; Schuh, H. Global Mapping Function (GMF): A new empirical mapping function based on numerical weather model data. Geophys. Res. Lett. 2006, 33, L07304. [CrossRef]

23. Wu, J.T.; Wu, S.C.; Hajj, G.A.; Bertiger, W.I. Effects of antenna orientation on GPS carrier phase. Astrodynamics 1992, 18, 1647-1660.

24. Petit, G.; Luzum, B. IERS conventions (2010) (No. IERS-TN-36). In Proceedings of the International earth rotation and reference systems service (IERS), Meudon, France, 17-19 September 2010.

25. Springer, T.A.; Beutler, G.; Rothacher, M. A New Solar Radiation Pressure Model for GPS Satellites. GPS Solut. 1999, 2, 50-62. [CrossRef]

26. Zhao, Q.; Dai, Z.; Wang, G.; Li, X.; Liu, J. Real-Time Precise BDS Clock Estimation with the Undifferenced Observation. Geomat. Inf. Sci. Wuhan Univ. 2016, 5, 686.

27. Riley, W.J.; Riley, W.J. Handbook of Frequency Stability Analysis; National Institute of Standards and Technology: Gaithersburg, MD, USA, 2008.

28. Sesia, I.; Tavella, P. Estimating the Allan variance in the presence of long periods of missing data and outliers. Metrologia 2008, 45, S134-S142. [CrossRef]

29. Wang, B.; Lou, Y.; Liu, J.; Zhao, Q.; Su, X. Analysis of BDS satellite clocks in orbit. GPS Solut. 2015, 20, 783-794. [CrossRef]

30. GLONASS Constellation Status (17-11-21). Available online: http://www2.unb.ca/gge/Resources/ GLONASSConstellationStatus.txt (accessed on 17 April 2018).

31. Hauschild, A.; Montenbruck, O.; Steigenberger, P. Short-term analysis of GNSS clocks. GPS Solut. 2012, 17, 295-307. [CrossRef]

32. Greengard, L.; Greengard, L.; Lee, J.-Y.; Lee, J.-Y. Accelerating the Nonuniform Fast Fourier Transform. SIAM Rev. 2004, 46, 443-454. [CrossRef]

33. Lee, J.-Y.; Lee, J.-Y.; Greengard, L.; Greengard, L. The type 3 nonuniform FFT and its applications. J. Comput. Phys. 2005, 206, 1-5. [CrossRef]

34. NUFFT (NFFT, USFFT) Software. Available online: https://cims.nyu.edu/cmcl/nufft/nufft.html (accessed on 17 October 2017).

(C) 2018 by the authors. Licensee MDPI, Basel, Switzerland. This article is an open access article distributed under the terms and conditions of the Creative Commons Attribution (CC BY) license (http://creativecommons.org/licenses/by/4.0/). 